\title{
WAVELENGTH-DEPENDENT TRIPLET STATE POPULATING MECHANISMS OF NAPHTHALENE IN HEAVY-ATOM HOSTS
}

\author{
D. SCHWEITZER *, J. ZUCLICH $\dagger$ and A.H. MAKI \\ Department of Chemistry, University of California, Riverside, California 92502, USA
}

Received 23 Octeber 1972

\begin{abstract}
We have made zero-field optically-cetected magnetic sesonance measurements on the triplet state of naphthalcne-d 8 incorporated in the heavy-atom host crystals p-dichlorobenzene, p-dibromobenzene, and s-tetrachlorobenzene. The dependence of the relative triplet sublevel populating rates on the exciting wavelength is interpreted in terms of the electronic excitation transfer mechanisms. We find that upon excitation of the host $S_{1} \leftarrow S_{0}$ transition in $p$-dichlorobenzene, energy transfer to the guest $T_{1}$ takes place via the guest $S_{1}$, whereas in the other host crystals tine intermediate is mainly the host $T_{1}$ band. Upon host $T_{1}-S_{0}$ excitation, trappins takes place from a spin-aligned exciton band in s-tetrachlorobenzene, whereas the initial alignment is lost prior to trapping in the dihalobenzene hosts.
\end{abstract}

\section{Introduction}

In recent years, the method of optically detected magnetic resonance (ODMR) of phosphorescent triplet states [ $1-4]$ has been extensively used for measuring the kinetic parameters of individual sublevels [5-11]. In particular, the deternination of the relative populating rate constants of tre triplet sublevels gives important inforration about the selective intersystem crossing pathways in isclated molecules. In this communication, we seport on the use of relative populating rate constants of tine triplet sublevels to distinguish between different triplet populating mechanisms. The system which we will focus on most closely is naphthalene- $d_{8}$ dissolved in a single crystal of 1,4-dichlorobenzene (DCB). The ordering of the energies is $T_{1}(G)<T_{1}(H)<S_{1}(G)<S_{1}(H)$, where $G$ is the guest, and $H$ is the host. In this system, depending upon the exciting wavelength, it is possible to populate $T_{1}(G)$ either by energy transfer from the host $T_{1}$ band, or by internal intersystem crossing.

\footnotetext{
* Permanent address: Max Planck Institute, Depariment of Molecular Physics; Heidelberg, Germany.

t U.S. Public Health Service Postdoctoral Fellow.
}

These two mechanisms are expected to lead to differing relative populating rates of the sublevels of $T_{1}(G)$. Having established different populating patterns from the two populating mechanisms, it is then possible to assess the principal populating mechanism of $T_{1}(G)$ which results from direct excitation of the host singlez, $S_{1}(H)$. In this case, the possibilities are $S_{1}(H) \rightarrow T_{1}(H) \rightarrow T_{1}(G)$, and $S_{1}(H) \rightarrow S_{1}(G) \rightarrow T_{1}(G)$. We find that the latter mechanism dominates in our samples of naphthalene- $d_{8}$ in $p$-dichlorobenzene, while the former mechanism appears more important for naph thalene- $d_{8}$ in $p$-dibromobenzene (DBB), and in s-tetrachlorobenzene (TCB). We also observe that spin alignment of the triplet excitons in TCB is maintained during the time interval required for trapping, whereas spin alignment is lost in the triplet exciton bands of the $p$-dihalobenzenes prior to trapping.

\section{Experimental}

All materials were purified by extensive zone refining (200-300 passes) and single crystals were grown by the Bridgman method. Samples were mounted in a helical slow-wave structure which terminates a coaxial microwave transmission line and is immersed 
in a liquid helium dewar kept at ca. $1.3^{\circ} \mathrm{K}$ by pumping. The sample was optically pumped either continu ously using a PEK, Inc. $100 \mathrm{~W}$ high pressure $\mathrm{Hg}$ lamp filtered by a $1 / 4$ meter Bausch \& Lomb monochromator, or using flash excitation from a PEK, Inc. $\mathrm{X}-80$ xenon flasilamp filtered with the appropriate interference filter. The 0,0 band of the naphthalene phosphorescence was selected with a McPherson, Inc. Model 2051 one meter grating monochromator fitted with a cooled EMI, Inc. Model $9558 \mathrm{QA}$ photomultiplier. The measurement of relative populating rate constants using microwave-induced phosphorescence transients during cw optical pumping has been described previcusly [10] as has their measurement using flash excitation methods [11]. All measurements were made in the absence of an externally applied magnetic field.

\section{Results and discussion}

The relative populating rates of the individual triplet sublevels of naphthalene- $d_{8}$ in DCB resulting from cw excitation and flash excitation methods are summarized in table 1 . In the cw excitation method [10], the relative populating rates are calculated using the observed decay constants of the individual triplet sublevels (which will be reported in a forthcoming communication [12]) assuming that spinlattice relaxation (SLR) effects are absent. The relative steady-state populations, however, are given correctly by this method even if SLR affects the decay constants. On the other hand, the flash excitation method [11] gives the relative populating rate con. stants correctly even in the presence of SLR. The steady-state populations are calculated assuming negligible SLR. A comparison of the steady-state populations arising from $310 \mathrm{~nm} \mathrm{cw}$ excitation with those calculated from flash excitation (assuming negligible SLR) shows that significant $\mathrm{T}_{z} \leftrightarrow \mathrm{T}_{y}$ relaxation occurs at $1.3^{\circ} \mathrm{K}$. The striking result, however, is the strong dependence of the relative populating rate constants on the exciting wavelength. Excitation at $340 \mathrm{~nm}$ (host $\mathrm{T}_{1} \leftarrow \mathrm{S}_{0}$ ) leads to approximately equal populating rates of the guest triplet sublevels, whereas excitation at $310 \mathrm{~nm}$ (guest $S_{1} \leftarrow S_{0}$ ) and at $280 \mathrm{~nm}$ (host $S_{1} \leftarrow S_{0}$ ) leads to experimentally indistinguishable results with $p_{y}>p_{z}>p_{x}$. Any sig. uificant competition of the energy transfer process $\mathrm{S}_{1}(\mathrm{H}) \rightarrow \mathrm{T}_{1}(\mathrm{H}) \rightarrow \mathrm{T}_{1}(\mathrm{G})$ with the predominant process, $S_{1}(H) \rightarrow S_{1}(G) \rightarrow T_{1}(G)$, would have resulted in distinguishable rates using $310 \mathrm{~nm}$ and $280 \mathrm{~nm}$ ex. citation.

We have made $\mathrm{cw}$ measurements on naphthalene- $d_{8}$ dissolved in DBB, and in TCB single cry'stal hosts. In the DBB host we find no excitation wavelength dependence of the steady-state populations of the triplet sublevels. We find $p_{x}: p_{y}: p_{z} \approx 4: 3: 3$, although these values which are obtained from cw measure. ments are undoubtedly influenced by SLR. The naphthalene $S_{1} \leftarrow S_{0}$ could not be excited in DBB due to the intense overlapping host $T_{1} \leftarrow S_{0}$ absorption. Since excitation of the host $S_{1} \leftarrow S_{0}$ transition leads to the same steady-state naph thalene sublevel populations as does excitation of the host $T_{1} \leftarrow S_{0}$ transition, it is reasonable to assume that energy transfer to the guest occurs mainly by the route, $S_{1}(H)$ $\rightarrow \mathrm{T}_{1}(\mathrm{H}) \rightarrow \mathrm{T}_{1}(\mathrm{G})$

Excitation of the host $T_{1} \leftarrow S_{0}$ transition $(330 \mathrm{~nm})$ in TCB doped with naphthalene- $d_{8}$ gives the relative

Table 1

Relative populating rates and steady-state populations of naphthalene-d 8 tripleí sublevels in $p$-dichloroberzene ${ }^{3}$ )

\begin{tabular}{|c|c|c|c|c|c|c|}
\hline \multirow{2}{*}{$\left.\lambda_{e}^{b}\right)$ c. $(\mathrm{nm})$} & \multicolumn{3}{|c|}{ Steady-state populations (\%) } & \multicolumn{3}{|c|}{ Populating rates (\%) } \\
\hline & $n_{x}^{0}$ & $n ?$ & $n_{z}^{0}$ & $D_{x}$ & $p_{y}$ & $p_{z}$ \\
\hline $340(\mathrm{cw})$ & 12.4 & 55.4 & 32.2 & 31.7 & 32.1 & 36.2 \\
\hline $\begin{array}{l}310(\mathrm{cw}) \\
280(\mathrm{cw})\end{array}$ & 4.6 & 64.2 & 31.5 & 14.2 & 44.2 & 41.6 \\
\hline 310 (flash) & 4.4 & 73.4 & 22.2 & 14.4 & 54.1 & 31.5 \\
\hline
\end{tabular}

a) Principal axes of naphthalene are: $x$ - out-of-plane, $y$ - in-plane long axis; b) for cw measurements, $n_{u}^{0}$ are correct while $p_{u}$ are inferred assuming negligible SLR; for flash measurements, $p_{u}$ are coriect, while $n_{u}^{0}$ are inferred assuming negligible SLR. 
populating rates $p_{x}: p_{y}: p_{z} \approx 1: 1: 4$ of the guest triplet sublevels, whereas excitation at $314.5 \mathrm{~nm}$ (primarily guest $\mathrm{S}_{1} \leftarrow \mathrm{S}_{0}$ ) leads to $p_{x}: p_{y}: p_{z} \approx 1: 5: 6$. Excitation at $280 \mathrm{~nm}$ (host $S_{1} \leftarrow S_{0}$ ) results in populating rates which are qualitatively in agreement with those found for excitation at $330 \mathrm{~nm}$, which is in contrast with the results of the 1,4dichlorobenzene host. It can be concluded that energy tronsfer to the triplet state of naphthalene in the TCB host occurs to a significant extent via $S_{1}(H) \rightarrow T_{1}(H) \rightarrow T_{1}(G)$. TCB is an example of a 1-dimensional exciton crystal [13] in that the strongly interacting molecules are translationally equivalent. Since the triplet energy propagates via an exchange interaction primarily between equivalent molecules, the loss of spin alignnent with respect to the molecular axis system is expected to be ineffi. cient *. It is interestins to note that in TCB doped with naphthaiene, $65-70 \%$ of the energy transfer from the triplet exciton band occurs to the $T_{z}$ sublevel, indicating that tranping occurs more rapidly than the loss of spin alignment of the exciton $\dagger$. Since the naphthalene concentration is estimated as $\approx 0 . \dot{5} \%$, the triplet exciton can move over at least 100 sites (along a chain) while retaining spin alignment. These results are in contrast with those found for the 1,4

* Francis and Harris [14] have abserved the retention of spin alignment in the TCB triplet exciton band by observing tile effect of saturating the exciton magnetic resonance on the $y$-trap emission.

The pieference of the populating rate for the $T_{2}$ sublevel of naphthalene in TCB is surprising in light of some recently reported work on TCB assigning the orbital symmetry of $T_{1}$ as ${ }^{3} B_{14}[15]$. We would expect then that the $T_{y}$ sublevel of TCB is mainly responsible for the electronic $T_{1} \leftarrow S_{0}$ absorption intensity. Since our crystals slow no exciton phosphorescence, the alignment of the exciton triplet should prefer $T_{y}$; the corresponding spin sublevel of naphthalene should have the highest populating tate. There are several possible explunations for this discrepancy: (i) the naphthalene axes may be rotated ie?a sive to TCB; (ii) the TCB $T_{1} \leftarrow S_{0}$ absorption could have considerable $b_{3 g}$ vibronic activity, which would result in $T_{z}$ alignment; (iii) trapping might occur preferentially to guest molecules in afjacent chains; and (iv) excitation at $330 \mathrm{~nm}$ could possibly be mainly $T_{2} \leftarrow S_{0}$, where $T_{2}$ might reasonably be ${ }^{3} B_{2 u}$, resultirg in $T_{z}$ activity (the origin of $T_{1}$ is at $\approx 370 \mathrm{~nm}$ ). Furher experiments are in progress to help clarify this point. dihalobenzene hosts in which considerable exchange interaction between translationally non-equivalent sites is expected $\boldsymbol{t}^{\dagger}$.

\section{Acknowledgement}

This research was supported by grants from the National Institutes of Health, and the National Science Foundation.

T Cheng et al. [16] have reported also that the populating rates of naphthalene-d sublevels in DBB are approximately equal indicating that the DBB spin alignmont is relaxed prior to zrapping.

\section{References}

[1] M. Sharnoff, J. Chem. Phys, 46 (1967) 3263.

[2] A.L. Kwiram, Chem. Fhys, Letters 1 (1967) 272.

[3] J. Schmidt and J.H. van der Waals, Chem. Phys. Letters 2 (1968) 640.

[4] C.B. Harris, D.S. Tinti, M.A. El-Sayed and A.H. Maki, Chem. Phys. Letters 4 (1969) 409 .

[5] 1. Schmidt, W.S. Veeman and J.H. van der Waals, Chem. Phys. Letters 4 (1969) 341.

16] J. Schnidt, D.A. Antheunis and J.H. van der Waals, Mol. Pinys, 22 (1971) 1.

17] D.S. Tinti and M.A. El-Sayed, J. Chem. Phys. 54 (1971) 2529.

[8] C.B. Harris, J. Chem. Phys. 54 (1971) 972.

[9] C.B. Harris and R.J. Hoover, Chem. Phys. Letters 12 (1971) 75

[10] C.J. Winscom and A.H. Maki, Chem. Phys. Letters 12 (1971) 264

[11] D. Schweitzer, J. Zuclich and A.H. Maki, Mol. Phys., to be published.

[12] D. Schweitzer, J. Zuclich and A.H. Maki, to be published.

[13] R.M. Hochstrasser and J.D. Whiteman, Organic scintillators and liguid scintillation counting, eds. D.L. Horrocks and C.T. Peng (Academic Press, New York, 1971) p. 511.

[14] A.H. Francis and C.B. Harris, J. Chem. Phys, 55 (1971) 3595 .

(15) A.H. Francis and C.B. Harris, J. Chem. Phys. 57 (1972) 1050.

[16] T.H. Cheng, N. Hirota and S.W. Mao, Chem. Phys. Letters 15 (1972) 274. 